\title{
Top quark and electroweak physics
}

\author{
Louise Skinnari ${ }^{* \dagger}$ \\ Cornell University (US) \\ E-mail: louise.skinnari@cern.ch
}

\begin{abstract}
Measurements of top quark and electroweak processes are central in confronting the Standard Model in all corners of phase space and probing signs of physics beyond this successful, yet incomplete, model. These proceedings summarize the status of physics involving the top quark and electroweak sectors, ranging from precision measurements of production cross sections and Standard Model parameters, such as the weak mixing angle, to studies of rare processes, such as electroweak vector-boson scattering and four top quark production. Latest results are presented from the ATLAS, CMS, and LHCb experiments at the Large Hadron Collider, along with two new legacy measurements from the CDF/D0 experiments at the Tevatron.
\end{abstract}

The 39th International Conference on High Energy Physics (ICHEP2018)

4-11 July, 2018

Seoul, Korea

\footnotetext{
*Speaker.

${ }^{\dagger}$ Presenting results from ATLAS, CMS, LHCb, CDF/D0.
} 


\section{Introduction}

Measurements of top quark and electroweak processes are essential in testing the Standard Model (SM) of particle physics. The SM has been studied at collider experiments for decades, and repeatedly held up to precision tests since its initial formulation in 1970s. Yet, the model is known to be incomplete, e.g. in failing to provide a dark matter candidate. Top quark and electroweak precision measurements are central in improving the modeling of collider data and tuning Monte Carlo (MC) event generators, constraining parton distribution functions (PDFs), extracting key SM measurements, etc. With high center-of-mass energy and large available datasets, experiments are additionally confronting the SM in challenging and previously inaccessible regions of phase space as well as probing very rare processes.

Top quark and electroweak measurements are also fundamental in the search program for new physics contributions beyond the SM. Precision measurements can both help constrain - or observe - new physics processes, e.g. enhancements of extremely rare production processes or processes involving anomalous couplings. Top quark, single- and multi-boson production processes are also dominant background processes in many direct new physics searches and Higgs boson measurements. These proceedings will discuss recent results within the top quark and electroweak sectors as presented during ICHEP2018 from the CERN Large Hadron Collider (LHC) and Tevatron experiments.

\section{Electroweak measurements}

Single $W / Z / \gamma$ boson production provides high-statistics samples from which to extract SM parameters and to test the self-consistency of the model. This includes e.g. $W$ boson mass measurements and extraction of the weak mixing angle from Drell-Yan events. The production of multiple bosons form a sensitive probe of beyond SM (BSM) gauge interactions. Analyses include measuring production cross sections, probing anomalous triple or quartic gauge couplings (aTGC or QGC, respectively), and studying rare vector-boson scattering (VBS) processes.

Notably, measurements of single boson production at the LHC are now performed not only differentially or double-differentially as a function of kinematic variables, but even triple-differentially. The CMS experiment [1] recently measured triple-differential cross sections of $\gamma+$ jets production, sensitive to e.g. gluon PDFs [2]. The ATLAS experiment [3] measured triple-differential cross sections for Drell-Yan production, sensitive to, among others, PDFs and the weak mixing angle [4]. Also the LHCb experiment [5] studies single-boson production, with recent measurements of $Z \rightarrow \tau^{+} \tau^{-}$production in the forward region ${ }^{1}$, where comparison of the $\tau \tau$ channel with measurements in the $e e / \mu \mu$ decay channels are consistent with lepton-flavor universality [6].

\subsection{Effective leptonic weak mixing angle}

The effective leptonic weak mixing angle $\left(\sin ^{2} \theta_{\text {eff }}^{\text {lept }}\right)$ is a key SM parameter, with an approximate $3 \sigma$ tension between the most precise single-experiment measurements from LEP and SLD. At hadron colliders, $\sin ^{2} \theta_{\text {eff }}^{\text {lept }}$ can be extracted by exploiting the forward-backward asymmetry $\left(A_{\mathrm{FB}}\right)$ in

\footnotetext{
${ }^{1}$ The LHCb detector has a geometrical acceptance corresponding to the pseudorapidity region $2<\eta<5$.
} 
Drell-Yan $e e / \mu \mu$ events. Recent measurements of $\sin ^{2} \theta_{\text {eff }}^{\text {lept }}$ include results in the $\mu \mu$ channel from D0 [7], an updated Tevatron CDF/D0 combination [8], and new measurements from ATLAS [9] and CMS [10] using $20128 \mathrm{TeV}$ data in the $e e / \mu \mu$ channels. The measurement strategy involves fitting either the mass dependence (Tevatron), or the mass and rapidity dependence (CMS), of the observed $A_{\mathrm{FB}}$ to SM predictions as a function of $\sin ^{2} \theta_{\mathrm{eff}}^{\text {lept }}$. An example of the dependence of $A_{\mathrm{FB}}$ on the rapidity and mass of the dilepton system from CMS is shown in Fig. 1(a). ATLAS instead infers the value of $A_{\mathrm{FB}}$ from the $A_{4}$ angular coefficient ${ }^{2}$ in dilepton mass and rapidity bins [9].

The results are summarized in Fig. 1(b). The Tevatron combination yields a measured value of $0.23148 \pm 0.00033$. Most precise is the ATLAS result, which benefits from a significant increase in sensitivity by including events with one forward electron $\left(2.5<|\eta|<4.9\right.$, where the $A_{\mathrm{FB}}$ variation with dilepton mass is largest, labeled $e e_{\mathrm{CF}}$ in Fig. 1(b)). Using central electrons and muons, ATLAS and CMS achieves similar precision. Including events with a forward electron, ATLAS measures $\sin ^{2} \theta_{\text {eff }}^{\text {lept }}=0.23140 \pm 0.0036$, nearing the precision of the Tevatron combination from one experiment alone. Data statistics and PDFs are the dominant systematic uncertainties.

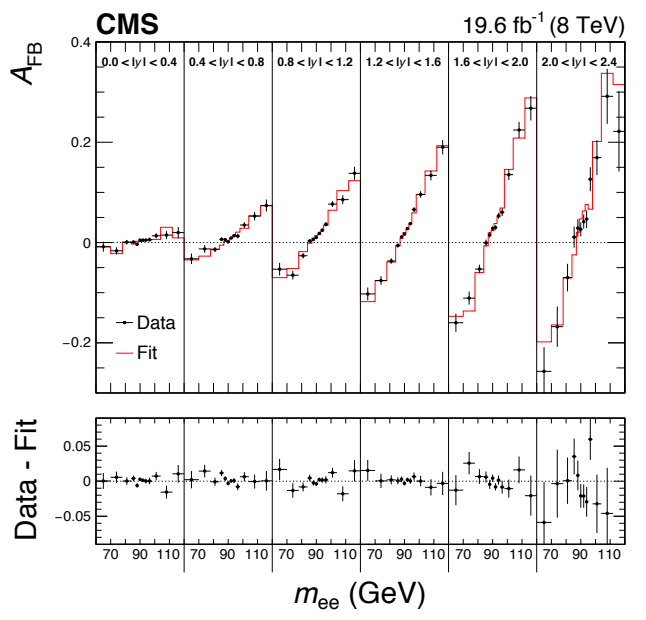

(a)

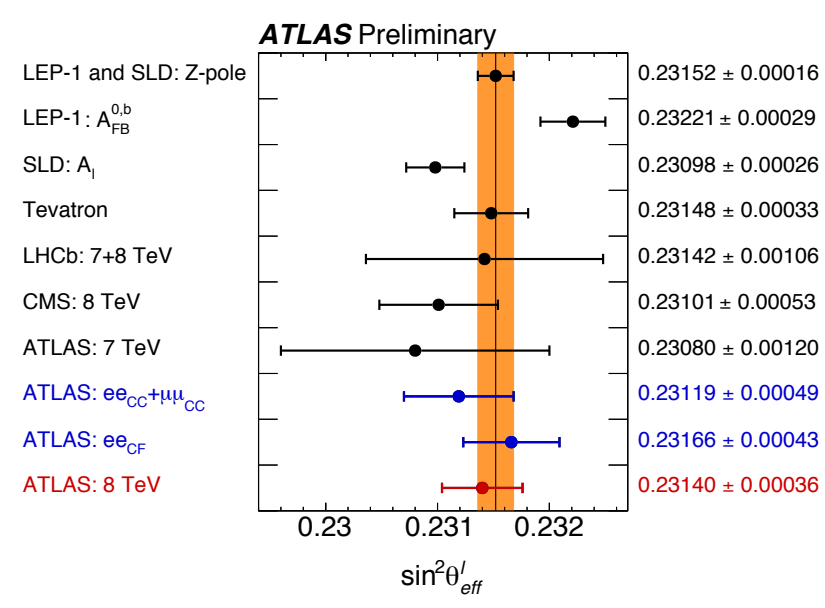

(b)

Figure 1: (1) Comparison between data and best-fit $A_{\mathrm{FB}}$ as a function of mass and rapidity in the dielectron channel at CMS [10]. (b) Overview of results from different measurements of $\sin ^{2} \theta_{\text {eff }}^{\text {lept }}[9]$.

\subsection{Diboson production $(Z Z / Z \gamma$ and $W Z)$}

$Z Z / Z \gamma$ production is sensitive to anomalous couplings (neutral aTGCs) that are forbidden in the SM and would thus be a clear sign of BSM interactions. SM ZZ production is also an important background process for Higgs boson measurements in the $H \rightarrow Z Z$ decay channel as well as to direct new physics searches. New inclusive and differential cross section measurements were presented for $Z Z$ production (ATLAS) [11] or ZZ production in association with jets (CMS) [12], in the $\ell^{+} \ell^{-} \ell^{\prime+} \ell^{\prime-}$ final state, using the $13 \mathrm{TeV} 2016$ datasets. ATLAS also measured the process $Z \gamma \rightarrow v v \gamma$ with the 2016 data [13]. These three analyses additionally searched for aTGCs, with no deviations observed and stringent limits placed.

\footnotetext{
${ }^{2} A_{4}$ is one the coefficients that together with the unpolarised cross section describe $Z$ boson production kinematics in perturbative quantum chromodynamics (QCD).
} 
ATLAS and CMS measured inclusive and differential cross sections for $W Z$ production in the $W Z \rightarrow \ell v \ell^{\prime+} \ell^{\prime-}$ decay mode with 2016 data $[14,15]$. Good agreement is observed between data and predictions. Several interpretations of the measurements are performed: CMS searches for and places stringent limits on aTGCs, and using lepton angular distributions, ATLAS measures the $W / Z$ boson polarization.

\subsection{Vector-boson scattering processes}

Processes involving scattering of massive vector bosons constitute important measurements in the study of electroweak symmetry breaking. In the SM, VBS scattering amplitudes are regulated by the Higgs boson and enhancements in the production rates of VBS processes would hint at contributions from BSM physics, such as a modified Higgs sector or the presence of new heavy resonances. VBS processes are also sensitive to anomalous QGC. Example Feynman diagrams of $W^{ \pm} W^{ \pm}, W^{ \pm} Z$, and $Z Z$ production through vector-boson scattering are shown in Fig. 2.
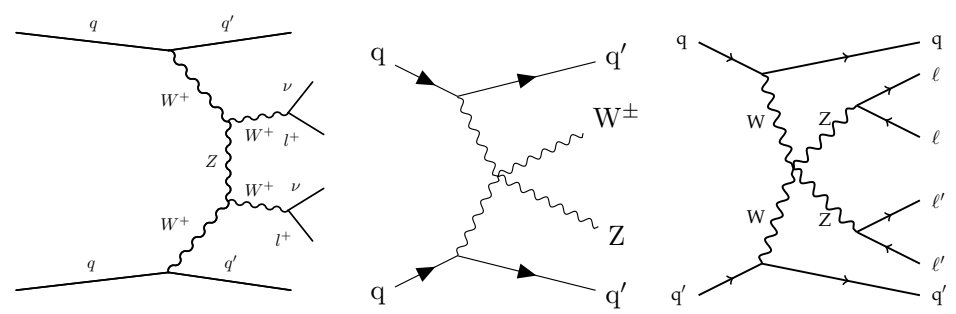

Figure 2: Example Feynman diagrams of electroweak $W^{ \pm} W^{ \pm}$(left), $W^{ \pm} Z$ (middle), and $Z Z$ (right) production through vector-boson scattering.

The characteristic signature of these electroweak production processes, which also distinguishes them from the QCD production process, is the presence of two jets with large rapidity separation and a high dijet mass. These properties were exploited to observe the rare $W^{ \pm} W^{ \pm}$process, first by CMS [16] and recently also by ATLAS [17] with an observed (expected) significance of $5.5 \sigma(5.7 \sigma)$ and $6.9 \sigma(4.6 \sigma)$, respectively. At ICHEP2018, first observation of electroweak $W^{ \pm} Z$ production was reported by ATLAS with $5.6 \sigma$ observed (3.3 $\sigma$ expected) significance [18], and a corresponding search presented from CMS with $1.9 \sigma$ observed (2.7 $\sigma$ expected) [19], the latter analysis also placing limits on aTGCs and charged Higgs bosons. Neither experiment has evidence for electroweak $Z Z j j$ production, though a search from CMS reaches an $2.7 \sigma$ excess (1.6 $\sigma$ expected) over the background-only hypothesis [20]. All analyses used the all-lepton final state and the $201613 \mathrm{TeV}$ datasets. Fig. 3 shows the dijet mass distribution for the CMS $W^{ \pm} W^{ \pm}$ measurement (3(a)), the sensitive variable, the output of a boosted-decision tree (BDT), from the ATLAS $W^{ \pm} Z$ measurement (3(b)), and the dijet mass used in the CMS ZZ search (3(c)).

\section{Top quark measurements}

The top quark has numerous unique properties: (i) it is the heaviest of all elementary particles and have a Yukawa coupling $y_{t} \sim 1$; (ii) it is extremely short-lived and decays before undergoing hadronization, resulting in that we can observe the properties of the bare quark; (iii) it is a window to new physics through both direct and indirect searches. 


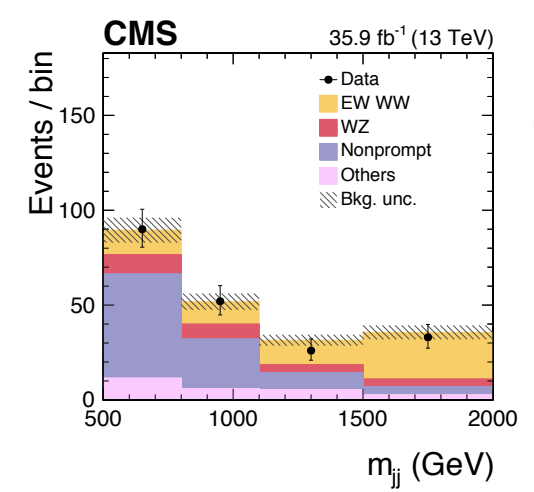

(a)

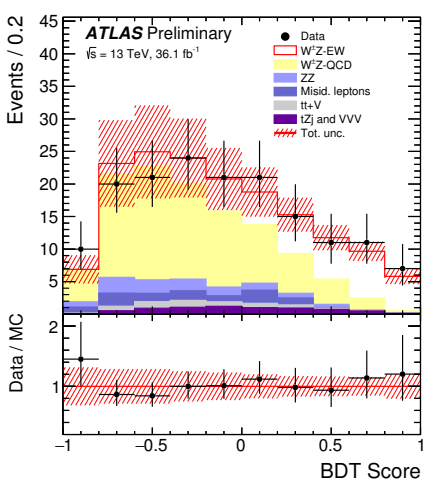

(b)

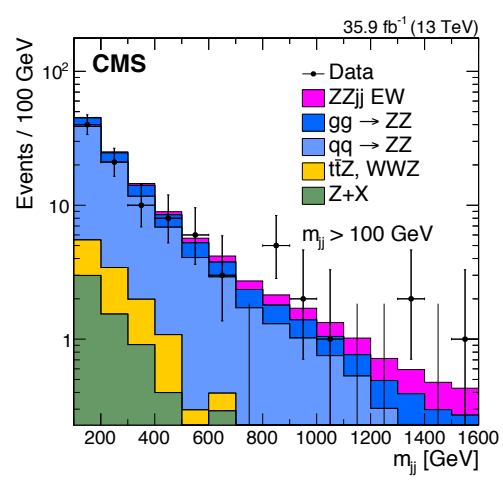

(c)

Figure 3: Dijet mass distribution for the CMS $W^{ \pm} W^{ \pm}$measurement [16] (left), BDT output from the ATLAS $W^{ \pm} Z$ measurement [18] (middle), and dijet mass from the CMS electroweak ZZ search [20] (right).

\subsection{Top quark production}

Top quarks can be produced in pairs, singly through the electroweak interaction, or in association with other particles, such as a $W / Z$ boson. Inclusive cross section measurements of top quark pair production $(t \bar{t})$ has been performed first at the Tevatron and more recently at the LHC at center-of-mass energies ranging between $5-13 \mathrm{TeV}$, as shown in Fig. 4. Measuring $t \bar{t}$ production tests QCD predictions and can be used to extract SM parameters and constrain top quarks as a background process. Good compatibility with theory predictions at high order in perturbation theory is observed, with the most precise measurements having a total uncertainty of 4-5\%, typically dominated by signal modeling and luminosity uncertainties [21, 22, 23].

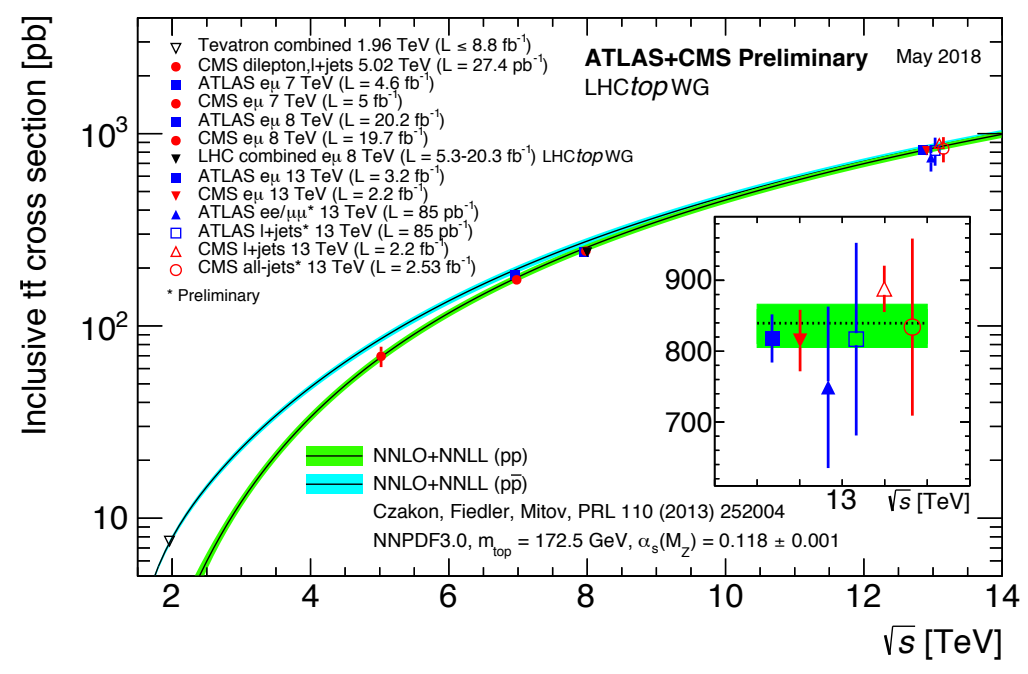

Figure 4: Inclusive $t \bar{t}$ production cross section as a function of center-of-mass energy, showing measurements from the Tevatron and the ATLAS and CMS experiments [24].

At LHC, electroweak (single) production of top quarks proceeds predominantly through either $t$-channel or $t W$ production, with $s$-channel production having only been observed at the Tevatron. 
The cross section extractions typically rely on multivariate techniques to distinguish signal from background. At ICHEP2018, a new measurement of the $t$-channel production mode using the 2016 $13 \mathrm{TeV}$ data was presented by CMS, measuring both the inclusive cross section and the ratio of top quark to anti-quark production, where the latter is particularly sensitive to PDFs [25]. Good agreement with SM predictions is observed.

ATLAS and CMS have recently reported evidence for rare single top quark production together with a $Z$ boson, $t Z$ production, with an observed significance of $4.2 \sigma$ and $3.7 \sigma$, respectively [26, 27]. The sensitive variable used in the ATLAS search, based on a neutral-network, is shown in Fig. 5(a). CMS has recently also reported first evidence for $t \gamma$ production, with an observed significance of $4.4 \sigma$ [28]. The sensitive variable, the output from a boosted decision tree, is shown in Fig. 5(b). All searches used the $201613 \mathrm{TeV}$ datasets, and show agreement with SM predictions.

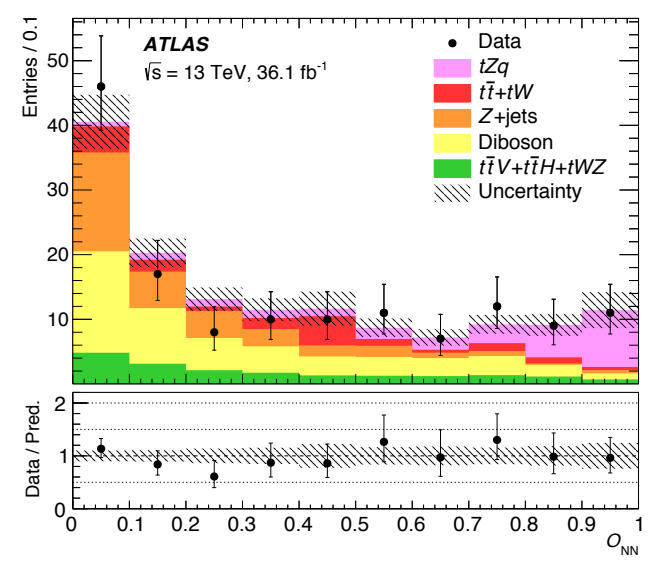

(a)

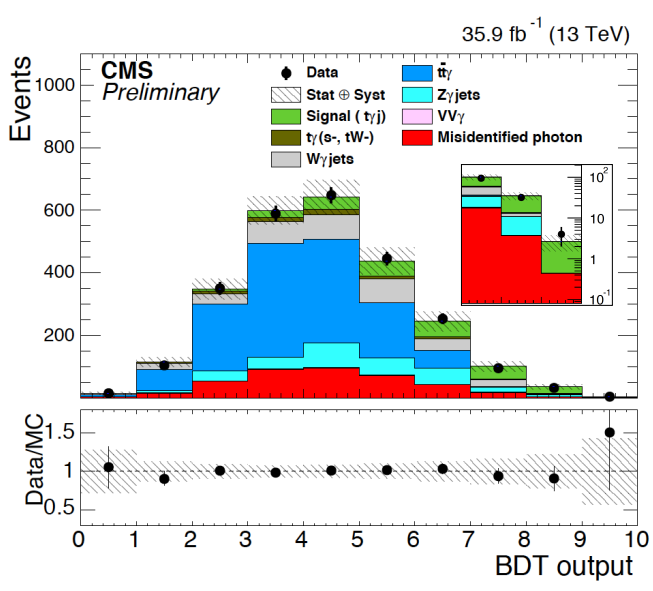

(b)

Figure 5: Sensitive variables in the ATLAS $t Z$ [26] (left) and CMS $t \gamma$ measurement [28] (right).

$t \bar{t}$ production has recently been measured at $\mathrm{LHCb}$ in the forward region $(2.0<\eta<4.5)$. The measurement, performed at $13 \mathrm{TeV}$, is statistics limited [29]. Top quarks have also been observed for the first time in proton-lead collisions by the CMS Collaboration [30]. It is a precise probe of the nuclear gluon density, and the measured result in consistent with predictions.

\section{Differential measurements}

Measuring differential top quark production cross sections as a function of different kinematic variables allows testing theory predictions, extracting SM parameters including the top quark mass and strong coupling $\alpha_{S}$, and to constrain PDFs. Differential measurements may also be sensitive to new physics effects. A recent analysis from CMS is shown in Fig. 6(a), where the normalized differential cross section in the dileptonic decay channel is presented as a function of the top quark transverse momentum $\left(p_{\mathrm{T}}\right)$ at $13 \mathrm{TeV}$ [31]. The measurement is compared to predictions from MC simulation and state-of-the-art of high-order QCD calculations. A mismodeling of the top quark $p_{\mathrm{T}}$ spectrum is observed, also seen at lower center-of-mass energies. The mismodeling is improved - but not solved - through the inclusion of electroweak corrections in the calculations. 
The modeling of $t \bar{t}$ production in association with additional heavy-flavor quarks is essential for $t \bar{t} H(H \rightarrow b \bar{b})$ measurements, where it is the dominant background process and its modeling constitute a large systematic uncertainty. Theoretical calculations of this process are challenging due to the massive $b$ quarks. ATLAS presented a new dedicated $t \bar{t} b \bar{b}$ analysis, measuring inclusive and differential cross sections [32]. Some discrepancies were observed, providing valuable input for MC tuning. An example is shown in Fig. 6(b) of the differential cross section as a function of invariant mass of the two $b$-jets closest in angular separation. The dominant uncertainty in the measurement is due to $b$-tagging and signal modeling.

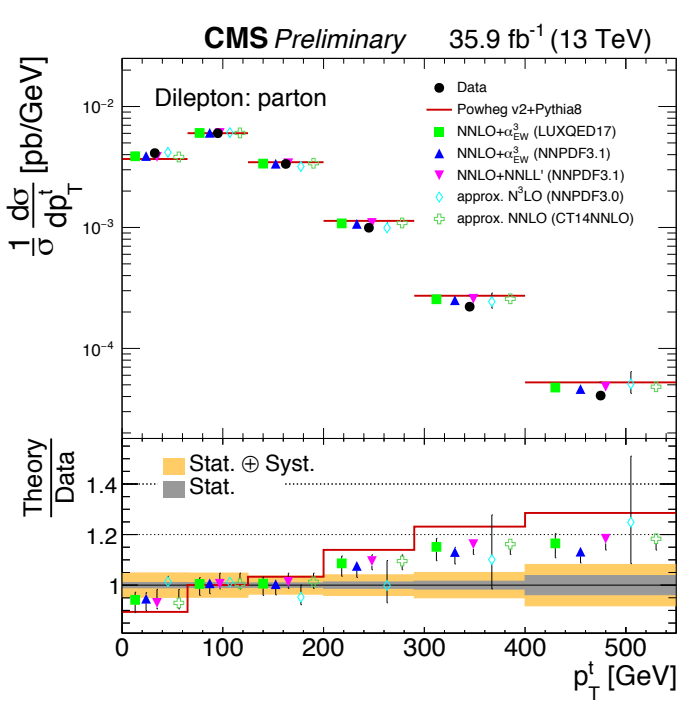

(a)

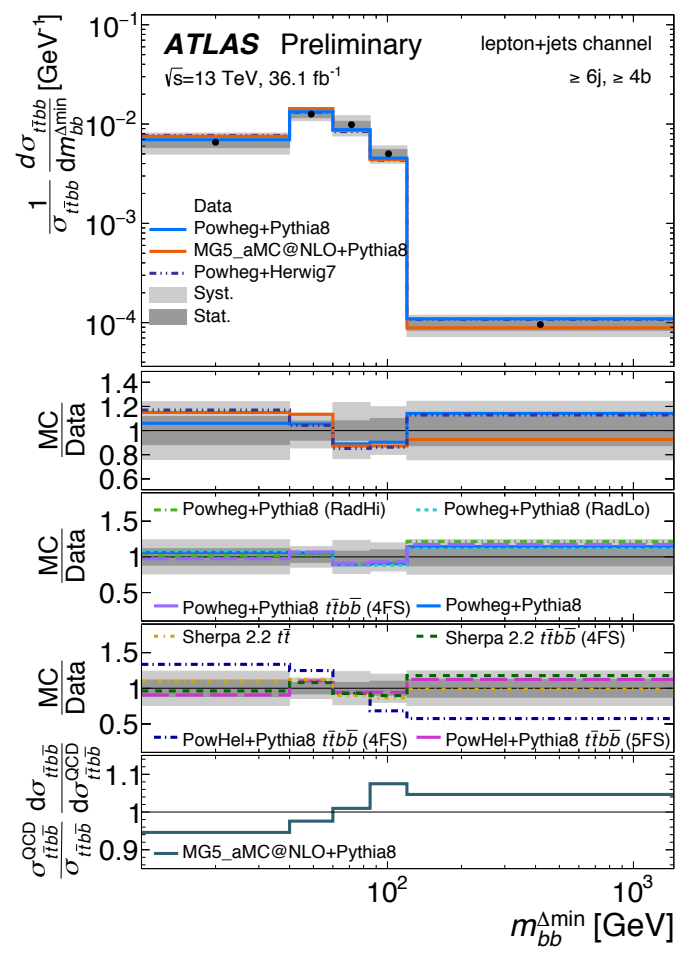

(b)

Figure 6: Normalized differential cross sections for (a) $t \bar{t}$ production as a function of top quark $p_{\mathrm{T}}$ in the dilepton channel from CMS [31] and (b) $t \bar{t} b \bar{b}$ production as a function of invariant mass of the two $b$-jets closest in angular separation from ATLAS [32] (right).

\subsection{Top quark properties}

Mass: Precise measurements of the top quark mass, an important SM parameter, are available from the Tevatron and LHC experiments. At ICHEP2018, a new measurement in the all-jets final state using the $201613 \mathrm{TeV}$ dataset was presented by CMS, determining the top quark mass and simultaneously constraining an additional jet energy scale factor (JSF). The measurement is $m_{\text {top }}=$ $172.34 \pm 0.20$ (stat + JSF) \pm 0.76 (syst) GeV [33]. 
Forward-backward asymmetry: A combination of the final CDF/D0 results of the forward-backward asymmetry for top quark pairs $\left(A_{\mathrm{FB}}(t \bar{t})\right)$, which may be altered through BSM interactions, was presented [34]. The measurements use the rapidity difference and resulting asymmetry between the decay leptons for fullyleptonic $t \bar{t}$ events. $A_{\mathrm{FB}}(t \bar{t})$ is measured in bins of $m(t \bar{t})$ and consistent with recent highprecision calculations (Fig. 7).

Spin correlations: The spin properties of top quarks are transferred to their decay leptons. These may be modified from the presence of a different top quark production mechanism or decay. Thus, measuring spin

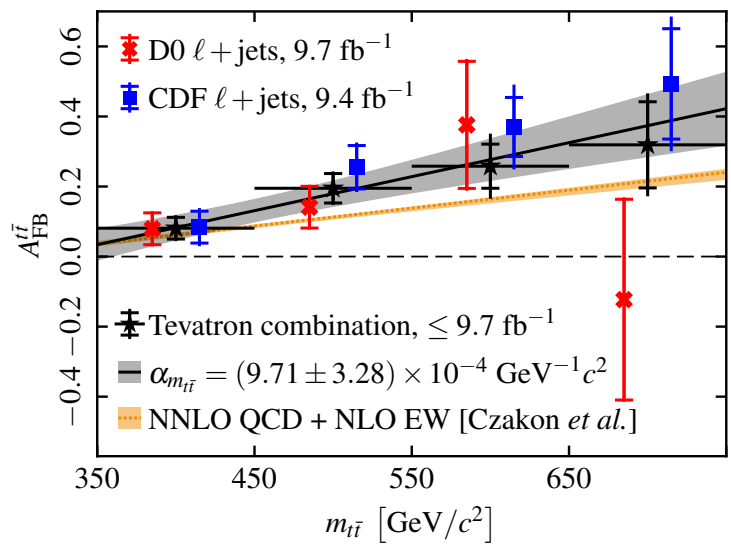

Figure 7: Tevatron measurements of the $t \bar{t}$ forwardbackward asymmetry as a function of the $t \bar{t}$ system mass, along with comparison to theory predictions [34]. correlations has sensitivity to new physics contributions. A new measurement in the $e \mu$ decay channel, with the $201613 \mathrm{TeV}$ data from ATLAS was presented [35]. Spin correlations are extracted from the normalized differential cross section, corrected to parton level, with respect to the difference in azimuthal angle between the leptons $\left(\mid \Delta \phi\left(\ell^{+} \ell^{-} \mid\right)\right.$, shown in Fig. 8(a). A 3.2 $\sigma$ discrepancy between the measurement and the prediction from simulations is observed.

Constraining new physics with EFTs: If the scale of new physics is significantly beyond that probed in direct BSM searches at the LHC, contributions from new physics due to higher-order operators can instead be constrained using an effective field theory (EFT) framework. A recent example is from CMS, where a measurement of the differential $t \bar{t}$ cross section in the dilepton channel as a function of the $\left|\Delta \phi\left(\ell^{+} \ell^{-}\right)\right|$is interpreted using ETFs [31]. Specifically, the analysis places constraints on the top chromo-magnetic dipole moment $\left(C_{t G}\right)$, shown in Fig. 8(b). The result is $-0.06<C_{t G} / \Lambda^{2}<0.41$, where $\Lambda$ is the assumed scale of the new physics, and corresponds to the most precise limits to date.

\subsection{Rare top quark processes}

SM production of four top quarks $(t \bar{t} t \bar{t})$ is an extremely rare process, yet to be experimentally confirmed. The predicted $t \bar{t} t \bar{t}$ cross section is about $10^{-5}$ times that of $t \bar{t}$ production at $13 \mathrm{TeV}$. Measurements of this process are sensitive to possible contributions from high mass scalars decaying to top quarks or modifications of the top Yukawa coupling. ATLAS and CMS have both searched for $t \bar{t} t \bar{t}$ production, most recently using the 2016 datasets. CMS uses the final state with a pair of same-sign or three leptons, with an observed (expected) signal significance of $1.6 \sigma(1.0 \sigma)$, and limits on $y_{t}$ [36]. ATLAS has searches in the same-sign and opposite-sign dilepton channels as well as the single lepton+jets final state, with a combined result of an observed (expected) excess over the background-only hypothesis of $2.8 \sigma(1.0 \sigma)$ [37]. With larger datasets on disk, pinning down this very rare process is now on the horizon. 


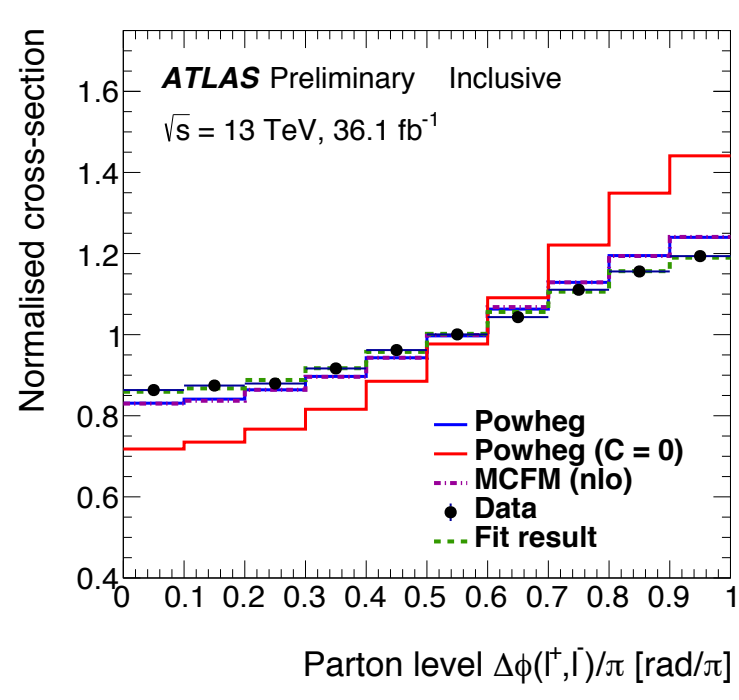

(a)

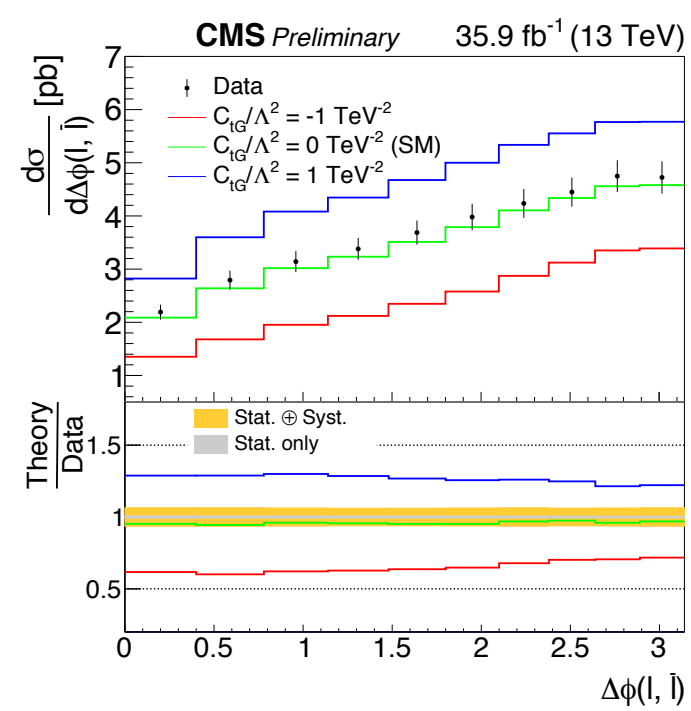

(b)

Figure 8: Measured normalized $t \bar{t}$ cross section as a function of $\left|\Delta \phi\left(\ell^{+} \ell^{-}\right)\right|$from ATLAS and predictions from POWHEG with (blue) or without (red) spin correlations as well as from MCFM including next-toleading order effects in top decays (dashed purple) and best-fit result (dashed green) [35] (left). Particle-level differential $t \bar{t}$ cross section as a function of $\left|\Delta \phi\left(\ell^{+} \ell^{-}\right)\right|$from CMS, comparing different values of $C_{t G} / \Lambda^{2}$ within an EFT framework [31] (right).

Other extremely rare top quark processes involve flavor-changing neutral currents (FCNC), which are highly suppressed in the SM. An observed enhancement of such decays would be a clear sign of BSM physics. ATLAS and CMS have performed numerous searches with different decay modes. The results are summarized in Fig. 9. In most scenarios considered, the searches are only beginning to approach sensitivity to various BSM models.

\section{Conclusions}

Measurements in the top quark and electroweak sectors are central in performing precision tests of the SM, pushing the limits of its validity, and searching for signs of new physics. Numerous new results were presented at ICHEP2018: legacy Tevatron combinations of the forward-backward asymmetry and leptonic weak mixing angle, LHC experiments pinning down the weak mixing angle to an, at the LHC, unprecedented precision, approaching the sensitivity of LEP and SLD. ATLAS and CMS are on the road to detailed vector-boson scattering studies with the observations of electroweak $W^{ \pm} W^{ \pm}$and $W^{ \pm} Z$ production. Precise top quarks results were presented, including some tantalizing discrepancies, and probing very rare SM processes. The results from the ATLAS and CMS experiments are based on data collected up to and including year 2016. Significantly larger datasets are on tape and expected through the end of LHC Run-2/3. Further on the horizon lies the upgrade to the HL-LHC and proposed lepton colliders, all that offer great potential for continued explorations of the top quark and electroweak sectors. 


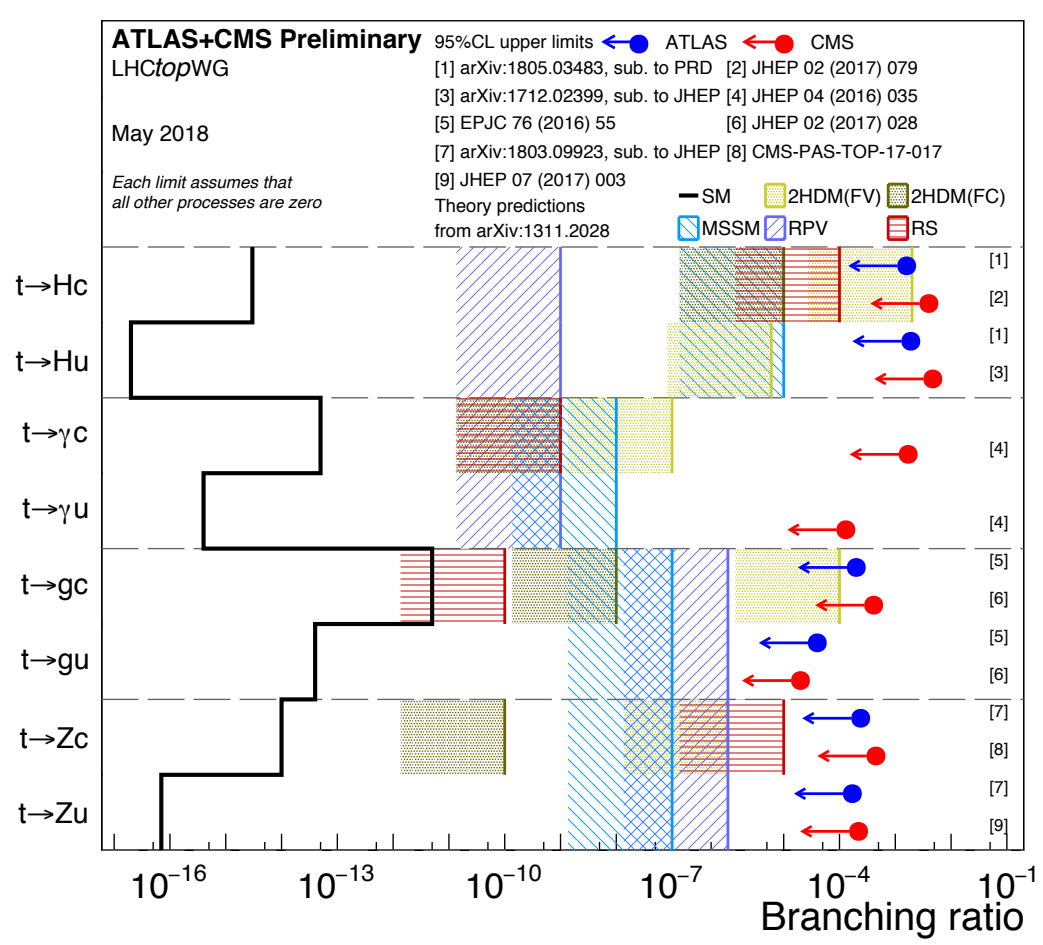

Figure 9: Limits from searches for FCNCs in different channels, with the SM prediction as well as enhancements expected from different BSM models [24].

\section{Acknowledgements}

This work was supported by the US National Science Foundation through NSF-PHY-1607126.

\section{References}

[1] CMS Collaboration, JINST 3 (2008) S08004.

[2] CMS Collaboration, Submitted to Eur. Phys. J. C.

[3] ATLAS Collaboration, JINST 3 (2008) S08003.

[4] ATLAS Collaboration, JHEP 12 (2017) 059.

[5] LHCb Collaboration, JINST 3 (2008) S08005.

[6] LHCb Collaboration, JHEP 1809 (2018) 159.

[7] D0 Collaboration, Phys. Rev. Lett. 120, 241802 (2018).

[8] CDF and D0 Collaborations, Phys. Rev. D 97, 112007 (2018)

[9] ATLAS Collaboration, ATLAS-CONF-2018-037 (2018).

[10] CMS Collaboration, Eur. Phys. J. C 78 (2018) 701.

[11] ATLAS Collaboration, Phys. Rev. D 97, 032005 (2018).

[12] CMS Collaboration, Submitted to Phys. Lett. B.

[13] ATLAS Collaboration, ATLAS-CONF-2018-035 (2018).

[14] ATLAS Collaboration, ATLAS-CONF-2018-034 (2018).

[15] CMS Collaboration, CMS-PAS-SMP-18-002 (2018).

[16] CMS Collaboration, Phys. Rev. Lett. 120, 081801 (2018).

[17] ATLAS Collaboration, ATLAS-CONF-2018-030 (2018).

[18] ATLAS Collaboration, ATLAS-CONF-2018-033 (2018).

[19] CMS Collaboration, CMS-PAS-SMP-18-001 (2018).
[20] CMS Collaboration, Phys. Lett. B 774 (2017) 683.

[21] ATLAS Collaboration, Phys. Lett. B761 (2016) 136.

[22] CMS Collaboration, Eur. Phys. J. C. 77 (2017) 172.

[23] CMS Collaboration, JHEP 09 (2017) 051.

[24] LHCTopWGSummaryPlots

[25] CMS Collaboration, CMS-PAS-TOP-17-011 (2018).

[26] ATLAS Collaboration, Phys. Lett. B 780 (2018) 557.

[27] CMS Collaboration, Phys. Lett. B 779 (2018) 358.

[28] CMS Collaboration, CMS-PAS-TOP-17-016 (2018).

[29] LHCb Collaboration, JHEP 1808 (2018) 174.

[30] CMS Collaboration, Phys. Rev. Lett. 119 (2017) 242001.

[31] CMS Collaboration, CMS-PAS-TOP-17-014 (2018).

[32] ATLAS Collaboration, ATLAS-CONF-2018-029 (2018).

[33] CMS Collaboration, CMS-PAS-TOP-17-008 (2018).

[34] CDF and D0 Collaborations, Phys. Rev. Lett. 120 (2018) 042001.

[35] ATLAS Collaboration, ATLAS-CONF-2018-027 (2018).

[36] CMS Collaboration, EPJC 78 (2018) 140.

[37] ATLAS Collaboration, paper in preparation. 